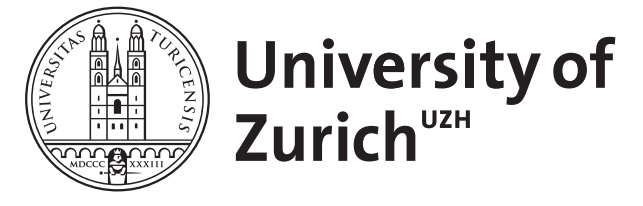

Zurich Open Repository and Archive

University of Zurich

University Library

Strickhofstrasse 39

CH-8057 Zurich

www.zora.uzh.ch

Year: 2016

\title{
Society Rules
}

Bernstein, Abraham

\begin{abstract}
Our society is full of rules: rules authorize us to achieve our goals by endowing us with legitimation, they provide the necessary structure to understand the chaos of conflicting indications or tell-tales of a situation, and oftentimes they legitimate our actions. But rules in society are different than logical rules suggest to be: they are not as unshakeable, continuously renegotiated, often even accepted to be wrong but still used, and used as inspiration in the situated context rather than universal truth. Based on theories about the role of technology in society, this talk will first try to convey the role of rules in social science theory. Extending these insights, it will draw on examples to illustrate how they might be transferred to computer science or artificial intelligence to derive systems that are attuned to the role of rules in social environments and adhere to social rules in the environment in which they are used.
\end{abstract}

DOI: https://doi.org/10.1007/978-3-319-45276-0

Posted at the Zurich Open Repository and Archive, University of Zurich ZORA URL: https://doi.org/10.5167/uzh-134971

Conference or Workshop Item

Originally published at:

Bernstein, Abraham (2016). Society Rules. In: 10th International Conference on Web Reasoning and Rule Systems (RR 2016), Aberdeen, 9 September 2016 - 11 September 2016, Springer.

DOI: https://doi.org/10.1007/978-3-319-45276-0 


\title{
Society Rules
}

\author{
Abraham Bernstein \\ University of Zurich, Zürich, Switzerland \\ Dynamic and Distributed Information Systems Group, Department of Informatics \\ bernstein@ifi.uzh.ch, \\ http://www.ifi.uzh.ch/ddis/people/bernstein.html
}

\begin{abstract}
Our society is full of rules: rules authorize us to achieve our goals by endowing us with legitimation, they provide the necessary structure to understand the chaos of conflicting indications or tell-tales of a situation, and oftentimes they legitimate our actions. But rules in society are different than logical rules suggest to be: they are not as unshakeable, continuously renegotiated, often even accepted to be wrong but still used, and used as inspiration in the situated context rather than universal truth.

Based on theories about the role of technology in society, this talk will first try to convey the role of rules in social science theory. Extending these insights, it will draw on examples to illustrate how they might be transferred to computer science or artificial intelligence to derive systems that are attuned to the role of rules in social environments and adhere to social rules in the environment in which they are used.
\end{abstract}

Keywords: Rules in the Social Realm, Non-standard reasoning, Adaptive Workflows, Specificity Frontier, Process Recombination, Cultural Adaptivity, Diverse and Accurate Recommendations

\section{Rules in Society}

Our Society gets governed by rules. Some are written explicitly such as laws; others are tacit and maintained by processes such as socialization or rites of passage [4]. Many of these rules are used very differently than in the canonical model often-times prescribed by logical rules. They change and evolve during actions [1], are only taken as indications rather than prescriptions for action [10], or are even completely ignored.

Despite this mismatch, the formalization of rules has led to incredible gains: Enterprise Ressource Planing Systems (ERPs) such a SAP enable the running of corporations, automated trading systems manage billions, fraud detection systems ensure the stability of our financial transactions. Some of these systems' properties have, however, prevented innovation, caused rigidity, and prevented adaptiveness due to an inability to deal with exceptions or lack of flexibility. In some cases, they may have even led to disasters, as they found themselves in situations that were not foreseen during design and implementation. 


\section{Social Rules in Systems}

Taking inspiration in social science theory about the role of rules and norms in society [6], this talks will explore examples of the loose interpretation of rules as the means for supporting the social rules, norms, or conventions. Each approach presented leverages the use of loosely or statistically specified and interpreted rules in the attempt of finding the sweet-spot between the efficiency of automated interpretation and flexibility of human activity.

The first example will explore an alternative view to process support or workflow management systems that provide flexibility. Based on a concept called the Specificity Frontier [2], it suggests that the relevant rules should be able to change during execution. This has recently lead to a system that interleaves the orchestration of crowds with auto-experimentation to determine the most appropriate process for a given task [3].

The second example will explore the elusive nature of cultural normsanother special set of societal rules - and how they can be leveraged to improve user interactions. Specifically, we show how a rule-based system paired with a very generalizing interpretation of insights from cultural anthropology allow to generate user interfaces that automatically adapt the users' cultural background. These generated user interfaces are shown to increase both the efficiency and effectiveness of users' interactions with the system [7-9].

Time permitting, the third example will take us to the realm of recommending TV shows, where we will see that also statistical reasoning needs to be "bent" to the social rules that govern this specific setting by foregoing recommendation accuracy in favor of diversity and speed [5].

\section{References}

1. Stepehen R. Barely. Technology as an occasion for structuring: Evidence from observations of ct scanners and the social order of radiology departments. Administrative Science Quarterly, 31(1):78-108, March 1986.

2. Abraham Bernstein. How can cooperative work tools support dynamic group process? bridging the specificity frontier. In Proceedings of the 2000 ACM Conference on Computer Supported Cooperative Work, CSCW '00, pages 279-288, New York, NY, USA, 2000. ACM.

3. Patrick M. De Boer and Abraham Bernstein. Pplib: Toward the automated generation of crowd computing programs using process recombination and autoexperimentation. ACM Trans. Intell. Syst. Technol., 7(4):49:1-49:20, April 2016.

4. John Seely Brown and Paul Duguid. Organizational learning and communities-ofpractice: Toward a unified view of working, learning, and innovation. Organization Science, 2(1):40-57, February 1991.

5. Fabian Christoffel, Bibek Paudel, Chris Newell, and Abraham Bernstein. Blockbusters and wallflowers: Accurate, diverse, and scalable recommendations with random walks. In Proceedings of the 9th ACM Conference on Recommender Systems, RecSys '15, pages 163-170, New York, NY, USA, 2015. ACM.

6. Wanda J Orlikowski. The duality of technology: Rethinking the concept of technology in organizations. Organization science, 3(3):398-427, 1992. 
7. Katharina Reinecke and Abraham Bernstein. Improving performance, perceived usability, and aesthetics with culturally adaptive user interfaces. ACM Trans. Comput.-Hum. Interact., 18(2):8:1-8:29, July 2011.

8. Katharina Reinecke and Abraham Bernstein. Knowing what a user likes: A design science approach to interfaces that automatically adapt to culture. MIS Quarterly, 37(2):427-453, 2013.

9. Katharina Reinecke, Minh Khoa Nguyen, Abraham Bernstein, Michael Näf, and Krzysztof Z. Gajos. Doodle around the world: Online scheduling behavior reflects cultural differences in time perception and group decision-making. In Proceedings of the 2013 Conference on Computer Supported Cooperative Work, CSCW '13, pages 45-54, New York, NY, USA, 2013. ACM.

10. Lucy A. Suchman. Plans and Situated Actions: The Problem of Human-machine Communication. Cambridge University Press, New York, NY, USA, 1987. 